\title{
Factores influyentes en la capacidad emprendedora de los alumnos de la Universidad Nacional Mayor de San Marcos
}

\begin{abstract}
RESUMEN
En el contexto de la globalización la necesidad de innovar, generar riqueza y desarrollar el potencial creativo del recurso humano se está enfatizando en el desarrollo del espiritu emprendedor.

El propósito de la presente investigación fue determinar los posibles factores que explicarían la capacidad de emprendimiento de los alumnos de la UNMSM, y a partir de ello contribuir a la formulación de los lineamientos que permitan a la Universidad desarrollar las bases educativas y formativas que fomenten la mentalidad empresarial de sus alumnos. Investigaciones anteriores habian establecido hasta tres factores para explicar la capacidad emprendedora en jóvenes universitarios; tomando como base estos antecedentes, y en la interacción del equipo multidisciplinario que condujo la investigación, se planteó la necesidad de incluir un cuarto factor, todo ello en base a los principios de la psicometría. Los resultados permitieron establecer cuatro factores que explican la capacidad de emprendimiento de jóvenes ingresantes a la UNMSM: capacidad de relacionarse socialmente, creatividad, capacidad de planificación y capacidad de realización personal. E cuarto factor considerado fue el de la creatividad.
\end{abstract}

Palabras clave: Emprendedor, capacidad de emprendimiento, jóvenes emprendedores.

DETERMINING FACTORS IN THE ENTERPRISING CAPACITY OF THE STUDENTS OF THE UNIVERSIDAD NACIONAL MAYOR DE SAN MARCOS

\section{ABSTRACT}

In the context of globalization the need to innovate, create wealth and develop the creative potential of human resources is emphasizing the development of entrepreneurship. The purpose of this investigation was to determine the possible factors that explain the abilities of students from San Marcos University and thus help in the formulation of guidelines to enable the University to develop educational and training bases to promote an enterprise culture of their students. Previous research had established three factors to explain entrepreneurship in university students, drawing primarily on this background and in the interaction of the multidisciplinary team that conducted the research, discussed the need to include a fourth factor, all based on the principles of psychometrics. The results indicate four factors that explain the abilities of young entrants to the UNMSM: ability to relate socially, creativity, capacity planning and realization. The fourth factor considered was that of creativity.

KEYWORDS: Entrepreneur, capacity venture, young entrepreneurs

\section{INTRODUCCIÓN}

El término de empresario aparece íntimamente unido al concepto de empresa concebida ésta como una realidad socioeconómica. El empresario personaliza la actuación de la empresa siendo la figura representativa que, según sus motivaciones, persigue unos objetivos coherentes con los fines a conseguir por la empresa en un intervalo temporal. En definitiva, el empresario se constituye como el órgano individual o colectivo encargado de establecer los objetivos empresariales y la toma de decisiones oportunas para alcanzarlos.

En la presente investigación entenderemos como emprendedor (entrepreneur, que a su vez viene de «inprendere» que significa acometer) a aquella persona que identifica una oportunidad y organiza los recursos necesarios para ponerla en marcha. Es común emplear este término para designar a una «persona que crea una empresa» o que encuentra una oportunidad de negocio, o a alguien quien empieza un proyecto por su propio entusiasmo.

Las investigaciones de percepciones describen al emprendedor con términos como innovador, flexible, dinámico, capaz de asumir riesgos, creativo y orientado al crecimiento. Los medios informativos, ya sea impresos, televisivos o virtuales ${ }^{4}$, a menudo definen el término emprendedor como la capacidad de iniciar y operar empresas nuevas. En todo caso ninguna definición del emprendimiento es lo suficientemente precisa o descriptiva para señalar a la persona o grupo (en sentido empresarial, social, investigativo o cualquier otro) que desea ser innovador, flexible y creativo.

Un emprendedor es aquel individuo que busca o ve una oportunidad y quiere o trata de convertirla en negocio, o simplemente busca nuevas formas para mejorar su vida y en algunos casos la de los demás; pero que no necesariamente es empresario.

\footnotetext{
Magíster en Construcción. Docente de la Facultad de Ingeniería Industrial de la UNMSM. danielmavila@yahoo.es

2 Magíster en Marketing y Turismo. Docente de la Facultad de Ingeniería Industrial de la UNMSM. otinocog@gmail.com

3 Ingeniero Insdustrial. Docente de la Facultad de Ingeniería Industrial de la UNMSM c_c_cesar@ yahoo.es

4 Suplemento diario La Primera. Microfinanzas. Setiembre 2009. dineba.minedu.gob.pe/xtras/ buen emprendedor.pdf
} 
Para que el emprendedor se convierta en empresario tiene que pasar el proceso que este último experimenta, debe pagar el precio que conlleva a convertirse en empresario, ya sea exitoso o no. Ser empresario implica una inteligencia emocional diferente, una forma de pensar diferente al resto, tiene que saber cómo operar sin recursos por largos periodos de tiempo, financieros generalmente. Todo esto tiene sustento en lo que Kantis (2008) denomina relación entre cultura y sistema educativo. Distintos aspectos culturales tales como la valoración social del emprendedor, las actitudes frente al riesgo de fracasar y la presencia de modelos de empresarios ejemplares constituyen factores culturales que inciden sobre la formación de vocaciones para emprender.

El empresario tiene que saber como tratar con diferentes tipos de personas, como construir equipos de trabajo, "como ser más efímero" (hacer cada vez más con cada vez menos).

El ser empresario es un proceso, donde hay fracasos, éxitos y más éxitos. Es en esta fase donde se quedan muchos emprendedores, ya que la inteligencia emocional que se necesita para superar los fracasos y aprender de los errores es indispensable.

Por lo que la espera al final del proceso de convertirse en empresario es cuando el emprendedor decide pagar por ese precio; la otra cara de la moneda, un mundo lleno de satisfacciones, la recompensa, es más que suficiente.

\section{DISEÑO EXPERIMENTAL}

La hipótesis planteada para la presente investigación fue "Existen diferencias en la capacidad emprendedora de los ingresantes de la UNMSM en relación al género, entorno familiar, ocupación de los padres, al tipo de institución educativa de procedencia y al tipo de preparación pre universitaria que recibieron".

Para elaborar la operacionalización de la variable se definió el concepto sobre la capacidad emprendedora para el caso de la presente investigación y luego se definieron las variables y los indicadores pertinentes para elaborar el tipo de preguntas que se elaboraron en el cuestionario aplicado a los estudiantes de la UNMSM.

La variable dependiente está dada por la capacidad emprendedora de los estudiantes de la UNMSM.
Variables independientes que se desprenden de la conceptualización de la variable dependiente:

1) Capacidad de realización. Está referida al impulso por vencer desafíos, avanzar y crecer, al esfuerzo por alcanzar el triunfo, metas y ser útiles a otros. Comprende:
A. La búsqueda de oportunidades
B La persistencia
C. La demanda por calidad y eficiencia.
D. La toma de riesgos.

2) Capacidad de planificación. Referida a pensar antes de actuar, identificando metas concretas o resultados que se desean alcanzar y diseñar planes consistentes para desempeñarse de manera que se aproveche al las oportunidades y anular o disminuir las amenazas del entorno. Comprende:

A. El establecimiento de metas.

B. La búsqueda de información, y

C. La planificación sistemática y el control.

3) Capacidad de relacionarse socialmente. Referida a la aptitud de generar estados de ánimo positivos en las personas de su entorno que se traduzcan en compromisos de apoyo para el desarrollo de sus proyectos o negocio. La persona capaz de establecer vínculos con personas e instituciones que aporten a su progreso. Se manifiesta a través de técnicas de comunicación efectiva. Comprende:
A. La persuasión y elaboración de redes de apoyo.

B. La autoconfianza.

4) Capacidad de creatividad. Referida a habilidad para sugerir soluciones y planteamientos creativos para obtener el éxito empresarial.

\section{MÉTODOS Y RESULTADOS}

Partiendo de investigaciones anteriores, (Gonzalez, 2003) y Tinoco (2008) que establecieron tres dimensiones para la medición de la habilidad emprendedora en alumnos universitarios, se consideró necesario ampliar las dimensiones a cuatro: realización personal, capacidad de relacionarse socialmente, planificación y creatividad, para lo cual se procedió a reelaborar el instrumento de medición, habiéndose considerado un cuestionario de 26 preguntas (Anexo 1).

Se recurrió a una muestra piloto de 90 estudiantes, con los cuales se verificaron dos aspectos: 
Primero, la prueba de confiabilidad, mediante el alfa de Cronbach, en el que se obtuvo un coeficiente de 0.923 , valor relativamente alto que denota la confiabilidad del instrumento. El valor mínimo de este coeficiente para medir confiabilidad es de 0.70. (Tamayo, 1981).
Segundo, la verificación de las dimensiones consideradas al elaborar el instrumento, lo cual se desarrolló mediante el análisis factorial, con soporte en el programa SPSS v. 15.0, y en el cual se obtuvieron las siguientes agrupaciones:

\section{Dimensión: Capacidad de relacionarse socialmente}

\begin{tabular}{|l|}
\hline P1. Identificas tus talentos y los usas para alcanzar tus propósitos \\
\hline P5. Propones nuevas alternativas para alcanzar tus propósitos \\
\hline P18. Trabajas cooperativamente en equipo \\
\hline P2. Actúas por iniciativa propia usando tus recursos \\
\hline P20. Construyes redes de apoyo \\
\hline P17. Comprendes y satisfaces las necesidades de tu interlocutor \\
\hline P13. Defines metas o propósitos concretos en tu desempeño \\
\hline P19. Influyes en los demás \\
\hline P7. Das soluciones fluidas y/o flexibles frente a los problemas \\
\hline P10. Mantienes vitalidad para desarrollar tus actividades \\
\hline P16. Evalúas y corriges las acciones \\
\hline P11. Cumples con los compromisos adquiridos \\
\hline
\end{tabular}

Dimensión: Capacidad de realización personal

$\mathrm{P}$ 15. Administras racionalmente los recursos

P21. Al enfrentarte a un problema consideras que es algo normal en la vida del ser humano.

P4. Atribuyes a ti mismo las causas y consecuencias de tus acciones

P14. Investigas, exploras, curioseas, preguntas

P23. De la solución a problemas pueden surgir nuevos caminos en tu vida.

\section{Dimensión: Creatividad}

P26. Crees que es posible dar usos nuevos a lo ya existente

P25. Te interesa saber cómo funcionan las cosas.

P24. Prestas atención a lo que otros denominan "ideas locas"

P22. Buscas que tus respuestas sean diferentes a la de los demás.

\section{Dimensión: Capacidad de Planificación}

P6. Visualizas anticipadamente el resultado de tus acciones

P3. Identificas, calculas y controlas los riesgos al emprender las acciones

P12. Aplicas controles de calidad (haces bien las cosas)

P9. Buscas perfeccionarte y superarte como persona

P8. Buscas y tomas oportunidades para resolver tus demandas o exigencias 
CUADRO 1: CARACTERÍSTICAS DE LA MUESTRA DE INGRESANTES 2009

\begin{tabular}{|l|c|c|c|}
\hline \multicolumn{1}{|c|}{ Facultad } & \multicolumn{2}{c|}{ Sexo } & Total \\
\hline Ingeniería Industrial & Masculino & Femenino & \\
\hline Ciencias Sociales & 57 & 33 & 90 \\
\hline Ciencias Administrativas & 28 & 44 & 72 \\
\hline Letras y Ciencias Humanas & 8 & 28 & 36 \\
\hline Ingeniería Geológica, Minas & 27 & 33 & 60 \\
\hline Ciencias Biológicas & 48 & 17 & 65 \\
\hline Ciencias Contables & 29 & 34 & 63 \\
\hline Total & 20 & 43 & 63 \\
\hline
\end{tabular}

CUADRO 2: ESTADÍSTICOS DESCRIPTIVOS PUNTAJE TOTAL

\begin{tabular}{|c|c|c|c|c|c|}
\hline $\mathrm{N}$ & Mínimo & Máximo & Media & Desv. típ. & C.V. \\
\hline 449 & 45 & 122 & 91,06 & 12,6 & $14 \%$ \\
\hline
\end{tabular}

FUENTE: Elaboración propia en base a datos recopilados.

CUADRO 3: ESTADÍSTICOS DESCRIPTIVOS PUNTAJE TOTAL SEGÚN FACULTAD

\begin{tabular}{|l|c|c|c|c|c|c|}
\hline \multicolumn{1}{|c|}{ Facultad } & N & Mínimo & Máximo & Media & Desv. típ. & CV \\
\hline Ingeniería Industrial & 90 & 73 & 122 & 95,50 & 12,10 & $13 \%$ \\
\hline Ciencias Sociales & 72 & 50 & 104 & 87,63 & 10,10 & $12 \%$ \\
\hline Ciencias Administrativas & 36 & 65 & 113 & 95,50 & 9,90 & $10 \%$ \\
\hline Letras y Ciencias Humanas & 60 & 53 & 112 & 89,05 & 13,96 & $16 \%$ \\
\hline Ingeniería Geológica, Minas & 65 & 45 & 114 & 87,74 & 14,49 & $17 \%$ \\
\hline Ciencias Biológicas & 63 & 55 & 106 & 88,27 & 10,94 & $12 \%$ \\
\hline Ciencias Contables & 63 & 50 & 120 & 94,24 & 12,44 & $13 \%$ \\
\hline Total & 449 & & & & & \\
\hline
\end{tabular}

Fuente: Elaboración propia en base a datos recopilados.

CUADRO 4: ESTADÍSTICOS DESCRIPTIVOS PUNTAJE TOTAL SEGÚN GÉNERO

\begin{tabular}{|l|c|c|c|c|c|c|}
\hline Género & $\mathrm{N}$ & Mínimo & Máximo & Media & Desv. típ. & CV \\
\hline Masculino & 217 & 45 & 120 & 90,70 & 12,97 & $14 \%$ \\
\hline Femenino & 232 & 45 & 122 & 91,40 & 12,26 & $13 \%$ \\
\hline Total & 449 & & & & & \\
\hline
\end{tabular}

Fuente: Elaboración propia en base a datos recopilados.

Luego, se procedió a aplicar el instrumento a los alumnos de la UNMSM comprendidos en la muestra final (Ver Cuadro 1).

\section{ANÁLISIS Y DISCUSIÓN}

Inicialmente se había considerado a los alumnos ingresantes de todas las facultades de la UNMSM, pero la muestra final quedó conforme se señala en el Cuadro 1:

El instrumento para medir la habilidad emprendedora de los alumnos ingresantes a la Universidad Nacional Mayor de San Marcos cuenta con 26 ítems, distribuidos en cuatro dimensiones: realización, relacionarse socialmente, creatividad y planificación. Los puntajes totales obtenidos en la muestra se detallan en el Cuadro 2.
De acuerdo al Cuadro 2, el promedio del puntaje total obtenido por los alumnos comprendidos en la muestra es 91,06 , lo cual denota una alta habilidad emprendedora; por otro lado, el coeficiente de variación (CV) indica una distribución homogénea de los mismos.

Considerando la facultad en donde estudian, el puntaje total alcanzado por los alumnos ingresantes 2009 considerados en la muestra presentan las siguientes características (Ver Cuadro 3).

Conforme al Cuadro 3, los puntajes más altos, en promedio, corresponden a alumnos de las facultades de Ingeniería Industrial y de Ciencias Administrativas $(95,50)$, seguidos por los de Ciencias Contables $(94,24)$. En todas las facultades consideradas, la distribución de los datos es homogé- 


\section{CUADRO 5: ESTADÍSTICOS DESCRIPTIVOS PUNTAJE TOTAL SEGÚN CONDICIÓN LABORAL}

\begin{tabular}{|l|c|c|c|c|c|c|}
\hline ¿Trabajas? & $\mathrm{N}$ & Mínimo & Máximo & Media & Desv. típ. & CV \\
\hline Sí, a tiempo completo & 12 & 77 & 114 & 95,00 & 12,63 & $13 \%$ \\
\hline Sí, a tiempo parcial & 44 & 70 & 122 & 94,48 & 12,28 & $13 \%$ \\
\hline Sí, ocasionalmente & 117 & 55 & 114 & 90,43 & 11,92 & $13 \%$ \\
\hline No & 276 & 45 & 120 & 90,61 & 12,87 & $14 \%$ \\
\hline
\end{tabular}

Fuente: Elaboración propia en base a datos recopilados.

CUADRO 6: ESTADÍSTICOS DESCRIPTIVOS PUNTAJE TOTAL SEGÚN INSTITUCIÓN EDUCATIVA EN DONDE TERMINARON LA EDUCACIÓN SECUNDARIA

\begin{tabular}{|l|c|c|c|c|c|c|}
\hline Institución Educativa & $\mathrm{N}$ & Mínimo & Máximo & Media & Desv. típ. & CV \\
\hline Estatal & 258 & 45 & 122 & 90,80 & 12,36 & $14 \%$ \\
\hline Parroquial & 31 & 50 & 120 & 91,26 & 14,49 & $16 \%$ \\
\hline Privada & 160 & 45 & 120 & 92,27 & 12,65 & $14 \%$ \\
\hline
\end{tabular}

Fuente: Elaboración propia en base a datos recopilados.

\section{CUADRO 7: ESTADÍSTICOS DESCRIPTIVOS SEGÚN DIMENSIONES DEL INSTRUMENTO DE} MEDICIÓN

\begin{tabular}{|l|c|c|c|c|c|c|c|}
\hline Dimensión & N & Mínimo & Máximo & Media & $\begin{array}{c}\text { Desv. } \\
\text { típ. }\end{array}$ & $\begin{array}{c}\% \text { respecto } \\
\text { puntaje ideal }\end{array}$ & $\begin{array}{c}\text { Puntaje } \\
\text { ideal }\end{array}$ \\
\hline Relacionarse socialmente & 449 & 18 & 57 & 41,16 & 6,82 & $69 \%$ & 60 \\
\hline Realización & 449 & 4 & 25 & 17,95 & 2,85 & $72 \%$ & 25 \\
\hline Creatividad & 449 & 6 & 20 & 14,05 & 2,44 & $70 \%$ & 20 \\
\hline Planificación & 449 & 2 & 25 & 17,90 & 3,23 & $72 \%$ & 25 \\
\hline
\end{tabular}

Fuente: Elaboración propia en base a dataos recopilados.

nea, de acuerdo al coeficiente de variación obtenido (CV).

En el Cuadro 4 se muestran los estadísticos de los puntajes totales según el género del alumno. En promedio, el puntaje total obtenido por las alumnas es ligeramente superior al de los varones, y respecto al coeficiente de variación, se observa una mayor homogeneidad en las alumnas.

En el Cuadro 5 se muestran los estadísticos de los puntajes totales según la condición laboral de los alumnos. En promedio, el puntaje total obtenido por alumnos que trabajan a tiempo completo es mayor que el de aquellos que laboran a tiempo parcial $u$ ocasionalmente y también con relación al de los que no trabajan. En todos los casos la distribución de los datos es homogénea.

De acuerdo al Cuadro 6, el promedio del puntaje total en la escala de habilidad emprendedora es mayor en aquellos estudiantes que terminaron la educación secundaria en entidades privadas, seguido del de las parroquiales y en tercer término de aquellos que lo hicieron en entidades del Estado. Resultados que corroboran los obtenidos por Tinoco (2008) en un estudio similar circunscrito a estudiantes de Ingeniería Industrial.

\section{Habilidad emprendedora según las dimensiones}

Considerando toda la muestra, se obtuvieron los siguientes resultados:

Según el Cuadro 7, las dimensiones en donde mejor se aproximan los puntajes de los alumnos al puntaje ideal corresponden a la realización personal y planificación.

\section{CONCLUSIONES}

a) Tomando como referencia el puntaje ideal en cada dimensión de la habilidad emprendedora y de acuerdo al Cuadro 8, los estudiantes de Ingeniería Industrial responden mejor, en creatividad y planificación; los de Ciencias Sociales en realización y planificación y los de Ciencias Administrativas en realización y planificación Los estudiantes de Letras y Ciencias Humanas respondieron mejor en realización y creatividad, mientras que los de Ingeniería Geológica lo hicieron en realización y planificación.

En Ciencias Biológicas los alumnos ingresantes respondieron mejor en las dimensiones de realización y planificación, al igual que los de Ciencias Contables. 


\section{CUADRO 8: ESTADÍSTICOS DESCRIPTIVOS SEGÚN DIMENSIONES Y SEGÚN FACULTAD}

\begin{tabular}{|c|c|c|c|c|c|c|}
\hline Facultad & Dimensión & $\mathrm{N}$ & Media & Desv. típ. & $\begin{array}{c}\% \\
\text { respecto } \\
\text { ptje ideal }\end{array}$ & $\begin{array}{c}\text { Puntaje } \\
\text { ideal }\end{array}$ \\
\hline \multirow{4}{*}{ Ingeniería Industrial } & Relacionarse socialmente & 90 & 42,53 & 6,81 & $71 \%$ & 60 \\
\hline & Realización & 90 & 18,59 & 2,80 & $74 \%$ & 25 \\
\hline & Creatividad & 90 & 15,69 & 2,19 & $78 \%$ & 20 \\
\hline & Planificación & 90 & 18,69 & 3,22 & $75 \%$ & 25 \\
\hline \multirow{4}{*}{ Ciencias Sociales } & Relacionarse socialmente & 72 & 40,08 & 5,64 & $67 \%$ & 60 \\
\hline & Realización & 72 & 17,11 & 2,31 & $68 \%$ & 25 \\
\hline & Creatividad & 72 & 13,01 & 1,57 & $65 \%$ & 20 \\
\hline & Planificación & 72 & 17,42 & 3,02 & $70 \%$ & 25 \\
\hline \multirow{4}{*}{ Ciencias Administrativas } & Relacionarse socialmente & 36 & 43,56 & 5,48 & $73 \%$ & 60 \\
\hline & Realización & 36 & 18,50 & 2,55 & $74 \%$ & 25 \\
\hline & Creatividad & 36 & 14,08 & 1,83 & $70 \%$ & 20 \\
\hline & Planificación & 36 & 19,36 & 2,50 & $77 \%$ & 25 \\
\hline \multirow{4}{*}{ Letras y Ciencias Humanas } & Relacionarse socialmente & 60 & 40,12 & 8,33 & $67 \%$ & 60 \\
\hline & Realización & 60 & 17,90 & 2,88 & $72 \%$ & 25 \\
\hline & Creatividad & 60 & 13,77 & 2,89 & $69 \%$ & 20 \\
\hline & Planificación & 60 & 17,27 & 3,64 & $69 \%$ & 25 \\
\hline \multirow{4}{*}{ Ingeniería Geológica, Minas } & Relacionarse socialmente & 65 & 40,03 & 7,32 & $67 \%$ & 60 \\
\hline & Realización & 65 & 17,20 & 3,24 & $69 \%$ & 25 \\
\hline & Creatividad & 65 & 13,62 & 2,67 & $68 \%$ & 20 \\
\hline & Planificación & 65 & 16,89 & 3,45 & $68 \%$ & 25 \\
\hline \multirow{4}{*}{ Ciencias Biológicas } & Relacionarse socialmente & 63 & 39,48 & 6,47 & $66 \%$ & 60 \\
\hline & Realización & 63 & 17,87 & 2,48 & $71 \%$ & 25 \\
\hline & Creatividad & 63 & 13,63 & 2,50 & $68 \%$ & 20 \\
\hline & Planificación & 63 & 17,29 & 2,64 & $69 \%$ & 25 \\
\hline \multirow{4}{*}{ Ciencias Contables } & Relacionarse socialmente & 63 & 42,90 & 6,09 & $72 \%$ & 60 \\
\hline & Realización & 63 & 18,56 & 3,22 & $74 \%$ & 25 \\
\hline & Creatividad & 63 & 14,02 & 2,12 & $70 \%$ & 20 \\
\hline & Planificación & 63 & 18,76 & 3,14 & $75 \%$ & 25 \\
\hline
\end{tabular}

Fuente: Elaboración propia en base a datos recopilados.

b) Por los resultados encontrados se puede afirmar que tenemos el capital humano potencial que tiene que ser mostrado al país, por lo que es importante que la motivación se logre trabajando con la persona, el cambio se debe generar desde la persona misma, para luego cambiar el liderazgo empresarial y por ende la visión de los negocios.

\section{RECOMENDACIONES}

a) Además de enseñar cursos relacionados a las finanzas y administración se deben efectuar talleres de motivación.

b) La Universidad tiene un reto para lograr que la brecha entre lo académico y lo empresario sea cada vez más corta.

c) Las facultades de la Universidad deben fortalecer el espíritu de empresa de los alumnos el cual se entenderá como la habilidad de la per- sona para transformar las ideas en actos. Esta competencia se relaciona con la creatividad, la innovación y la asunción de riesgos, así como con la habilidad para planificar y gestionar proyectos con el fin de alcanzar objetivos.

\section{REFERENCIAS BIBLIOGRÁFICAS}

Colectivo Integral de Desarrollo (2008). Programa emprendedores dinámicos. http://www.cid.org. pe/HomePage/index.htm (Visitado el 15 - 01 2009)

Comisión de las Comunidades Europeas. Bruselas, 13.2.2006 COM (2006) 33 final. Fomentar la mentalidad empresarial mediante la educación y la formación. http://www.juntadeandalucia.es/ averroes/ emprender/index. php?option $=$ com content\&task $=$ view\&id $=169 \&$ Itemid $=26$. Visitado 12-1-2009 
García Zapata, T. (2001). La universidad y su compromiso con las PYMES. Revista Industrial Data Vol. $4 \mathrm{~N}^{\circ}$ 2. pp 11-17.

Gonzales Gonzales, G. (2003). Capacidad emprendedora en estudiantes ingresantes de la UIGV. Tesis de Maestría.

Kantis, H. (2008). Emprendedores de origen humilde: ¿cómo incide la estructura social en la creación de empresas en América Latina? Banco Interamericano de Desarrollo.

FUNDES Internacional. Washington D.C. (Visitado 17-10-2009).

Kantis, H. Masahiko, I. Masahiko, K. (2002). Empresarialidad en economías emergentes: Creación y desarrollo de nuevas empresas en América Latina y el Este de Asia. http://www.iadb. org/sds/publication/publication_2934_s.htm. Banco Interamericano de Desarrollo. (Visitado 14-1-2009).
Inche J., Sandoval C. y Quispe C. (1998). Implementación de incubadora de negocios agroindustriales en la Facultad de Ingeniería Industrial de la UNMSM. Revista Industrial Data. Vol. $1 \mathrm{~N}^{\circ}$ 1: pp 15-17.

Pontificia Universidad Católica del Perú (2008). Centro de Innovación y Desarrollo Empresarial. http://www.pucp.edu.pe/cide/ (Visitado el 15-12009).

Tamayo, M. (1981). El proceso de la investigación científica. Editorial Limusa. México.

Tinoco G., O. (2008). Medición de la capacidad emprendedora de ingresantes a la Facultad de Ingeniería Industrial de la U NMSM. Instituto de Investigación de la FII. Taller de Investigación. http://campusvirtualfii.unmsm.edu.pe/campus/

Universidad del Pacífico (2008). Programa emprendedores dinámicos. http://www.up.edu.pe (Visitado el 15-1-2009). 


\section{Anexo 1}

\section{HABILIDAD EMPRENDEDORA}

Estimado alumno, el siguiente cuestionario es parte de una investigación orientada a la determinación de los factores que influyen en la capacidad emprendedora con la finalidad de que en la UNMSM promocione la creación de negocios como parte del quehacer de todo futuro profesional. Te agradecemos por la valiosa colaboración que prestas a este estudio, y esperamos llenes el documento con objetividad.

\section{INFORMACIÓN GENERAL}

a. Mes y año de nacimiento 119
B. Sexo:
(1) Masculino
(2) Femenino

C. La Institución Educativa donde culminaste tu educación secundaria fue:
(1) Estatal
(2) Parroquial
(3) Privada

D. La zona donde resides actualmente, pertenece al distrito:
Trabajas:
(1) Sí, a tiempo completo
(2) Sí, a tiempo parcial
(3) Sí, ocasionalmente
No

EN LAS SIGUIENTES AFIRMACIONES MARCA UNA, Y SÓLO UNA, DE LAS OPCIONES INDICADAS A CONTINUACIÓN

\begin{tabular}{|c|c|c|c|c|c|}
\hline $\mathbf{0}$ & 1 & $\mathbf{2}$ & $\mathbf{3}$ & $\mathbf{4}$ & $\mathbf{5}$ \\
\hline \multirow{2}{*}{ Nunca } & Casi nunca & $\begin{array}{c}\text { En } \\
\text { ocasiones }\end{array}$ & $\begin{array}{c}\text { Con } \\
\text { frecuencia }\end{array}$ & $\begin{array}{c}\text { Casi } \\
\text { siempre }\end{array}$ & Siempre \\
\hline
\end{tabular}

1. Identificas tus talentos y los usas para alcanzar tus propósitos

2. Actúas por iniciativa propia usando tus recursos

3. Identificas, calculas y controlas los riesgos al emprender las acciones

4. Atribuyes a ti mismo las causas y consecuencias de tus acciones

5. Propones nuevas alternativas para alcanzar tus propósitos

6. Visualizas anticipadamente el resultado de tus acciones

7. Das soluciones fluidas $y / 0$ flexibles frente a los problemas

8. Buscas y tomas oportunidades para resolver tus demandas o exigencias

9. Buscas perfeccionarte y superarte como persona

10. Mantienes vitalidad para desarrollar tus actividades

11. Cumples con los compromisos adquiridos

12. Aplicas controles de calidad (haces bien las cosas)

13. Defines metas o propósitos concretos en tu desempeño

14. Investigas, exploras, curioseas, preguntas

15. Administras racionalmente los recursos

16. Evalúas y corriges las acciones

17. Comprendes y satisfaces las necesidades de tu interlocutor

18. Trabajas cooperativamente en equipo

19. Influyes en los demás

20. Construyes redes de apoyo

21. Al enfrentarte a un problema consideras que es algo normal en la vida del ser humano.

22. Buscas que tus respuestas sean diferentes a la de los demás.

23. De la solución a problemas pueden surgir nuevos caminos en tu vida.

24. Prestas atención a lo que otros denominan "ideas locas"

25. Te interesa saber cómo funcionan las cosas.

26. Crees que es posible dar usos nuevos a lo ya existente 